\title{
Early management of parotid gland injury with oral nortriptyline and closed drain
}

\author{
Chan Min Chung', \\ Sung Jae Wee, \\ Hyoseob Lim ${ }^{1}$, \\ Sang Hun Cho', \\ Jong Wook Lee ${ }^{2}$ \\ ${ }^{1}$ Department of Plastic and \\ Reconstructive Surgery, Hallym \\ University Sacred Heart Hospital, \\ Hallym University College of Medicine, \\ Anyang; ${ }^{2}$ Department of Plastic and \\ Reconstructive Surgery, Hallym \\ University Hangang Sacred Heart \\ Hospital, Seoul, Korea
}

Parotid gland plays the most critical role in saliva secretion in the oral cavity. Parotid gland injuries due to facial trauma can cause various complications such as formation of a fistula or sialocele. Thus, such saliva-related complications can interfere with wound healing and increase the risk of infection. Several previous studies have discussed the treatment of fistula or sialocele. Nonetheless, prevention of such complications is of utmost importance. We present a case of parotid gland injury due to trauma to the cheeks that was surgically treated, with early postoperative management involving oral administration of nortriptyline and closed drainage, without complications.

Keywords: Antidepressive agent, tricyclic / Drainage / Nortriptyline / Salivary gland / Wound, stab

\section{INTRODUCTION}

Of the three paired major salivary glands, the parotid gland is the largest one and plays the most critical role in saliva secretion in the oral cavity [1]. The main causes of parotid injury include penetrating wounds, perforation wounds, and secondary injuries due to surgical procedures (e.g., parotidectomy) [2]. Stevenson [3] demonstrated injuries to the parotid gland area in facial trauma by dividing it into three regions. Based on masseter, those in regions anterior and overlying parts of the masseter were each associated with complications of about $80 \%$, such as facial nerve injury, sialocele, and salivary fistula [4]. In such cases, the continuously produced saliva is collected inside the wound, which leads to secondary complications, including infection. Thus, suppression of saliva production and proper drainage during the early postoperative management are im-

\footnotetext{
Correspondence: Hyoseob Lim

Department of Plastic and Reconstructive Surgery, Hallym University Sacred Heart Hospital, Hallym University College of Medicine, 22 Gwanpyeong-ro

170beon-gil, Dongan-gu, Anyang 14068, Korea

E-mail: hyoseob.lim@gmail.com

Received December 10, 2019 / Revised July 15, 2020 / Accepted August 18, 2020
}

portant.

Hereby, we report the case of a 79-year-old female patient with parotid gland injuries who underwent early postoperative management through oral administration of nortriptyline accompanied by a relatively long-term maintenance of closed drainage.

\section{CASE REPORT}

A 79-year-old woman visited our emergency department with three stab wounds in her right preauricular area (Fig. 1). We found no abnormal finding during the retrograde irrigation test for Stensen duct that was performed in the ampulla. However, facial expressions such as the area around the mouth and blinking of the eyes were unnatural, so the possibility of facial nerve injury could not be ruled out. An emergency exploration was performed under general anesthesia in the operating room. To minimize additional injuries and conduct a more reliable exploration of the injured areas, the wounds were connected to expose all the injured areas. Even though more dead space was created due to this procedure, we definitely found injuries in 


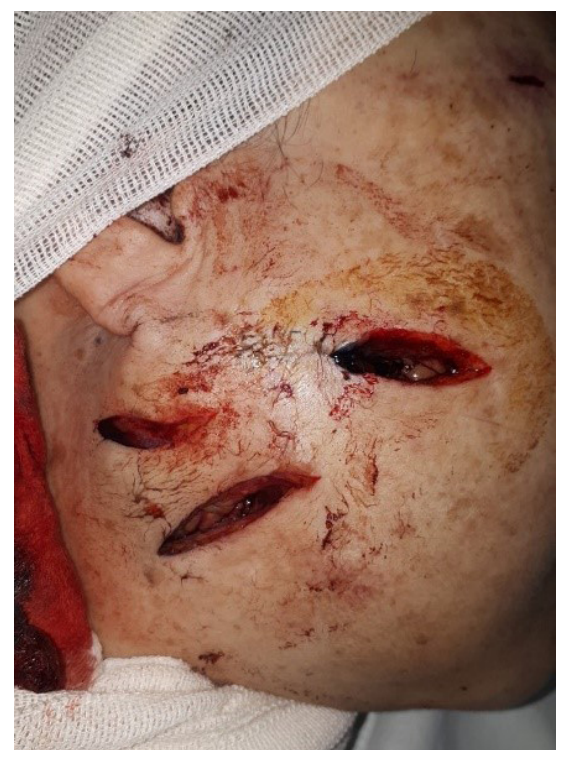

Fig. 1. A 79-year-old woman with three stab wounds on the right cheek.

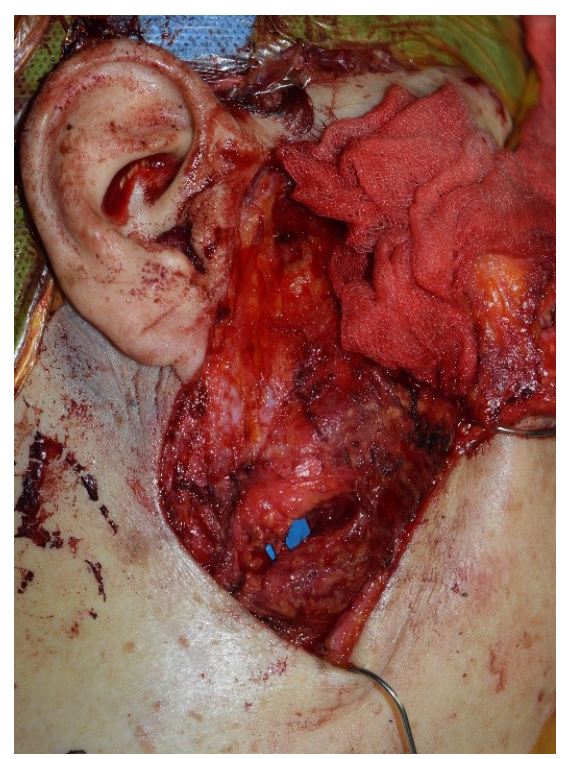

Fig. 2. Intraoperative photograph showing repaired marginal mandibular branch of the facial nerve and injured parotid gland.

the parotid glands and an injury at a marginal mandible branch of the facial nerve (Fig. 2). After neurorrhaphy for the facial nerve injury, the wound was sutured, and a closed $100-\mathrm{mL}$ Jackson-Pratt drainage tube was inserted (Fig. 3). The injured parotid gland area was not wide in size and the tissue was soft, so it was not repaired.

As postoperative management, oral administration of $10 \mathrm{mg}$ nortriptyline once daily for 3 weeks was started from the day of surgery to prevent secondary complication, such as infection due to persistent salivation. During treatment with nortripty-

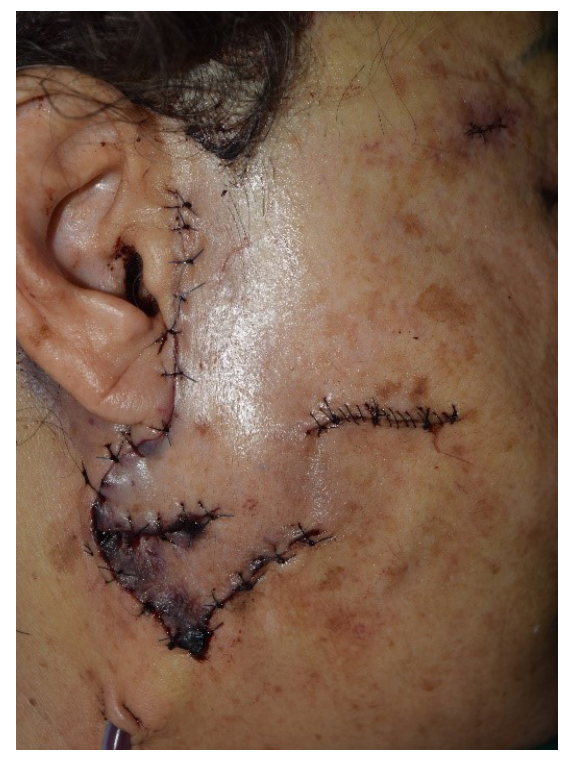

Fig. 3. Postoperative photograph 1 day after surgery.

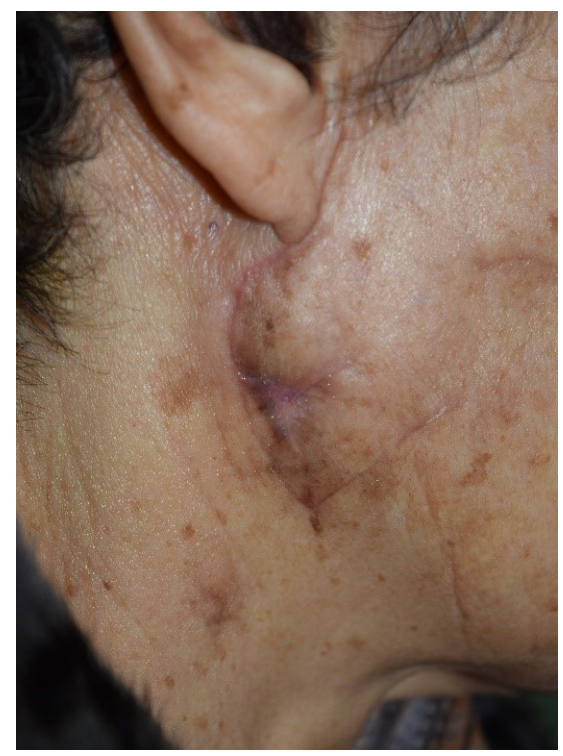

Fig. 4. Postoperative photograph 7 weeks after surgery.

line, the patient complained of mouth dryness, which showed that the drug had an anticholinergic action. The drainage tube was removed on the 6th day after the surgery, when $5 \mathrm{~mL}$ per day or less drainage was collected for 3 days in a row. Due to the possibility of partial wound dehiscence, delayed stitch out was planned, and all sutures were removed on the 10th postoperative day. Complete and unremarkable wound healing was observed, and no complications were reported 7 weeks postoperatively (Fig. 4). 


\section{DISCUSSION}

In this case, oral administration of nortriptyline combined with closed drainage for longer than usual were made to allow wound healing without complications. Nortriptyline is a tricyclic antidepressant, which was originally developed as an antidepressant and is known to have anticholinergic action as an adverse reaction. The anticholinergic action systemically suppresses secretion of serous fluids, causing cardiotoxicity, cognitive disturbance, confusion, seizure, blurred vision, dry mouth, urinary difficulty, and constipation [5,6]. Because of these side effects, tricyclic antidepressants are contraindicated in the event of severe impairment of myocardial performance, seizure disorders, glaucoma or large prostate [7]. When the salivary glands are injured, suppression of salivary secretion can promote wound healing and adhesion between wound surfaces. In the present case, we explained to patient and her guardians the legitimacy of using anticholinergic drug for suppression of salivary secretion and all possible aforementioned side effects. In addition, the appropriateness of the drug dose was assessed through consultation with the department of psychiatry and internal medicine twice a week.

No study has investigated the duration of administration for drugs with an anticholinergic effect in patients with injuries in the salivary glands. As an antidepressive agent, onset of therapeutic actions of nortriptyline usually sets in after 2 to 4 weeks. Treatment with antidepressant is symptomatic and should be monitored for a period of time [8]. In addition, according to the wound healing principle, maturation in the wounds starts at around 3 weeks after the injury via hemostasis, inflammation, and proliferation. Thus, we administered nortriptyline for 3 weeks postoperatively, which could be considered as the time point at which the duration of treatment as an anticholinergic agent and complete wound healing could be attained.

The closed drainage tube continuously provides negative pressure inside the wound, facilitating active drainage. Although this depends on the case, the drainage tube is usually removed when it drains less than $20 \mathrm{~mL}$ [9]. By contrast, this case involved injuries in the parotid glands inside the wounds, so the drainage tube was removed after the drainage amount was observed to be $5 \mathrm{~mL}$ per day for 3 days in a row. By maintaining the drainage longer, collection of liquid inside the wound was actively prevented. This also helped wound healing by maintaining contact between the wound surfaces. Closed drainage tubes generate negative pressure in the entire wound, although not significantly, which could additionally promote wound healing [10].

Once complications such as sialocele, fistula, and infection oc- cur in the wound, the wound becomes difficult to treat and, in some cases, could be fatal for the patient. Previous studies mostly described methods that included surgical drainage, simple conservative treatment, and parotid gland removal to treat such complications [11]. We showed that oral administration of nortriptyline and closed drainage could prevent such complications.

Previous studies also introduced other approaches to suppress secretion from the salivary glands, which included injection of botulinum toxin to the salivary glands and refraining from oral intake of foods. Vargas et al. [12] achieved a successful outcome of botulinum toxin injection in a patient with postoperative sialocele after incision of a portion of the parotid gland. However, because the facial nerve passes through the parotid gland, such a procedure may affect the nervous system function. Although the method to refrain from oral food intake may suppress secretion from the salivary gland effectively, obtaining consent from patients seems more difficult than drug administration. By contrast, in this case, obtaining consent from the patient to perform the treatment procedure is easier owing to the relatively lower risk it confers to the patient.

In our case, the injured parotid gland was treated without repair, partly because the injury was minor. However, theoretically, all salivary secretory cells are equipped with a salivary secretion system; thus, any injury to parotid gland or ducts, including Stensen duct, can cause the aforementioned complications. Various treatments have been performed because there is no consensus on the appropriate practice of this condition, from pressure dressing and needle aspiration to surgical approaches such as duct repair, ligation, and even parotidectomy $[13,14]$. Majority of authors seemed to agree that the treatment for parotid duct injuries is immediate repair using stents [3]. Moreover, Hills et al. [15] described a modified microsurgical parotid duct repair method, the Seldinger technique with a central venous catheter, which was suited to cannulate the parotid duct without iatrogenic perforation. Nonetheless, the other smaller ducts in the parotid glands, compared to the Stensen duct, are too small to repair. Studies have been conducted on the treatment of those unrepaired ducts. Parekh et al. [14] reported healing of minor intra-parotid duct injuries only by conservative therapy. Gordin et al. [2] examined 51 patients who developed delayed complications after parotid gland injury and observed that most of the patients responded to conservative therapy. These findings suggest that most salivary gland injuries undergo spontaneous repair even with conservative treatment alone, so they may require only close, though longer, monitoring for further complications.

Oral administration of nortriptyline combined with a rela- 
tively long maintenance of closed drainage can be significantly helpful for the healing of wounds accompanied by salivary gland injuries. However, further research with a large population is warranted. In addition, the amount of closed drainage and the removal criteria for the drainage tube should be further discussed. Information from an investigation of the appropriate duration of nortriptyline administration would also be useful.

\section{NOTES}

\section{Conflict of interest}

No potential conflict of interest relevant to this article was reported.

\section{Ethical approval}

The study was approved by the Institutional Review Board of Hallym Sacred Heart Hospital (IRB No. 2019-10-002) and performed in accordance with the principles of the Declaration of Helsinki. Written informed consent was obtained.

\section{Patient consent}

The patient provided written informed consent for the publication and the use of her images.

\section{ORCID}

Chan Min Chung

Sung Jae Wee

Hyoseob Lim

Sang Hun Cho

Jong Wook Lee

https://orcid.org/0000-0002-2755-9440

https://orcid.org/0000-0002-2397-7450

https://orcid.org/0000-0002-6251-8281

https://orcid.org/0000-0001-5906-9828

https://orcid.org/0000-0002-3244-949X

\section{REFERENCES}

1. Kochhar A, Larian B, Azizzadeh B. Facial nerve and parotid gland anatomy. Otolaryngol Clin North Am 2016;49:273-84.

2. Gordin EA, Daniero JJ, Krein H, Boon MS. Parotid gland trauma. Facial Plast Surg 2010;26:504-10.

3. Stevenson JH. Parotid duct transection associated with facial trauma: experience with 10 cases. Br J Plast Surg 1983;36:81-2.

4. Halse S, Rokke M, Bachmann-Harildstad G. Sialoceles of the parotid gland. Tidsskr Nor Laegeforen 2017;137:39.

5. Pomara N, Shao B, Choi SJ, Tun H, Suckow RF. Sex-related differences in nortriptyline-induced side-effects among depressed patients. Prog Neuropsychopharmacol Biol Psychiatry 2001;25: 1035-48.

6. Miller JJ, Whiting WL, Catalano G, Sanchez DL. Nortriptyline abuse: a case report and review of the literature. Clin Neuropharmacol 2019;42:108-10.

7. Williams GO. Management of depression in the elderly. Prim Care 1989;16:451-74.

8. Stephen M. Stahl. Prescriber's guide: antipsychotics: Stahl's essential psychopharmacology. 6th ed. New York: Cambridge; 2017.

9. Woo SH, Kim JP, Park JJ, Shim HS, Lee SH, Lee HJ, et al. Comparison of natural drainage group and negative drainage groups after total thyroidectomy: prospective randomized controlled study. Yonsei Med J 2013;54:204-8.

10. Kim PJ, Attinger CE, Steinberg JS, Evans KK. Negative pressure wound therapy with instillation: past, present, and future. Surg Technol Int 2015;26:51-6.

11. Akinbami BO. Traumatic diseases of parotid gland and sequalae: review of iterature and case reports. Niger J Clin Pract 2009; 12:212-5.

12. Vargas H, Galati LT, Parnes SM. A pilot study evaluating the treatment of postparotidectomy sialoceles with botulinum toxin type A. Arch Otolaryngol Head Neck Surg 2000;126:421-4.

13. Hong SE, Kwon JW, Kang SR, Park BY. The effect of botulinum toxin on an iatrogenic sialo-cutaneous fistula. Arch Craniofac Surg 2016;17:237-9.

14. Parekh D, Glezerson G, Stewart M, Esser J, Lawson HH. Posttraumatic parotid fistulae and sialoceles: a prospective study of conservative management in 51 cases. Ann Surg 1989;209:10511.

15. Hills AJ, Kannan RY, Williams M. Seldinger technique in repair of the parotid duct. Br J Oral Maxillofac Surg 2019;57:85-7. 